\title{
OXIDATIVE STRESS IN PERINATAL ASPHYXIA AND FETAL ASPHYCTIC PRECONDITIONING
}

\author{
K. Cox ${ }^{1,2,3}$, E. Strackx ${ }^{3}$, E. Vlassaks ${ }^{3}$, M. Sparnaaij ${ }^{3}$, L. Zimmermann ${ }^{1}$, J. Vles ${ }^{2}$, D. Gavilanes ${ }^{1}$ \\ ${ }^{1}$ Pediatrics, ${ }^{2}$ Child Neurology, ${ }^{3}$ Mental Health and Neuroscience, Maastricht UMC, Maastricht, The \\ Netherlands
}

Background: Asphyctic preconditioning is thought to attenuate the cellular stress response which reduces brain damage.

Objective: We evaluated the expression of stress related genes in pre- and neonatal pups after fetal preconditioning (FA) and perinatal asphyxia (PA).

Methods: FA was induced on E17 by clamping the uterine circulation for $30^{\prime}$. On P0 PA was induced by placing the fetuses-containing uterine horns in water for $19^{\prime}$. Control and FA pups were sacrificed 96h after the FA insult. Control, FA, PA and FA+PA pups were sacrificed 6h (P0) and 96h (P4) after birth. mRNA expression of iNos, nNos and Sod 1 was evaluated in the right hemisphere with qRT-PCR.

Results: Sod1 was significant down-regulated $(\mathrm{p}=0.0057)$ on $\mathrm{E} 21$ for the FA group compared to controls. On P4 both the PA and the FA+PA group were down-regulated when compared to controls $(p=0.0021$, $\mathrm{p}=0.0132)$ and compared to the FA group $(\mathrm{p}=0.0007, \mathrm{p}=0.0297)$. On $\mathrm{P} 4$ there was a significant difference between the PA and the FA+PA groups ( $p=0.0005)$ (fig.1). nNos expression revealed a significant increase on P0 for the PA and FA+PA groups compared to the FA group $(\mathrm{p}=0.0012, \mathrm{p}=0.0145)$. iNos showed no significant differences.

Conclusions: Modulation of the expression of antioxidants like Sod1 by FA could contribute to neuroprotection at birth. Additional whole-genome expression will provide a complete overview of the preconditioned phenotype.

\section{Sod1 expression on P4}

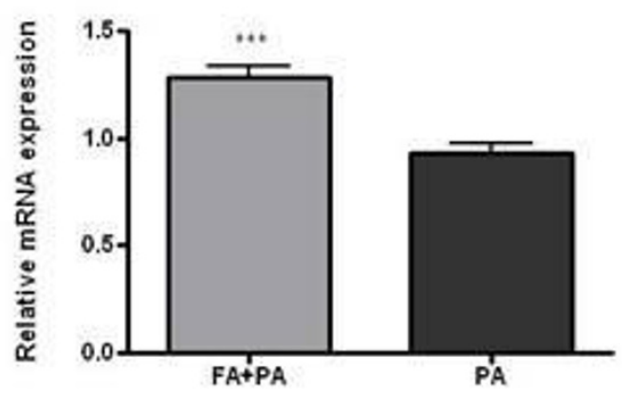

[fig, 1] 